programs featuring an intervention to remove unnecessary urinary catheters at a tertiary care center in Thailand. Infect Control Hosp Epidemiol 2007;28:791-798.

\section{A Formula for Infection Control Using Colonization Pressure and Compliance Rates}

To the Editor-Colonized and infected patients are a major reservoir for the patient-to-patient transmission of nosocomial pathogens within hospitals. The prevalence of colonized patients, termed "colonization pressure," is an important risk factor for the development of nosocomial infections. Colonization pressure is formally defined as the number of colonized patient-days $\times 100 /$ total patient-days. ${ }^{1}$ Bonten et $\mathrm{al}^{2}$ first described the role of colonization pressure in the spread of vancomycin-resistant enterococci (VRE) and showed that, when VRE colonization pressure increased threefold, from $25 \%$ to $75 \%$, the days until VRE acquisition decreased by one-third, from 16-18 days to 6-7 days. This indicates that, as colonization pressure increases threefold, the transmission velocity or power increases threefold. This group also reported that, when colonization pressure reached $50 \%$, it became an independent variable that determined VRE acquisition but was not affected by infection control measures, including handwashing and the wearing of personal protective equipment (PPE; eg, gloves, gowns and aprons, and masks). In methicillin-resistant Staphylococcus aureus (MRSA) nosocomial infections, the same relationship between the colonization pressure and the infection risk was observed. ${ }^{3}$ These findings suggested that low colonization pressure might reduce the likelihood of MRSA transmission even in settings with low rates of compliance to infection control measures, whereas high colonization pressure could result in transmission even in settings with high rates of compliance to infection control measures. Thus, a high colonization pressure may have the ability to negate infection control efficacy. ${ }^{4}$

This negation is thought to arise because the rate of compliance to infection control measures is generally assumed to be $30 \%-80 \%$, and reaching a $100 \%$ rate of compliance is difficult. ${ }^{5}$ Even at an $80 \%$ compliance rate, $20 \%$ of interactions are still noncompliant with infection control measures. If many carriers exist (ie, under high colonization pressure), the chance of direct contact (with carriers) and indirect contact (with the environment around carriers) increases, and the absolute number of noncompliant interactions also increases. However, low colonization pressure does not result in a high absolute number of potential contacts with infectious pathogens, even if the compliance rate is low. Overall, infection strength seems to be correlated with colonization pressure and inversely proportional to the rate of compliance to infection control measures.

Using the 2 variables of colonization pressure and compliance rate, transmission risk (ie, transmission velocity or transmission power) could simply be expressed as follows: transmission risk $=$ colonization pressure $(\%)$ of nosocomial pathogens/compliance rate (\%) of infection control measure. For instance, according to the formula, in the case of a low colonization pressure (eg, 10\%) and an ordinary compliance rate (eg, $40 \%$ ), the transmission risk can be calculated as $10 /$ $40=0.25$. However, in the case of a high colonization pressure (eg, 40\%) and a high compliance rate (eg, 80\%), the transmission risk can be calculated as $40 / 80=0.5$. Thus, even if the compliance rate is increased twofold and colonization is increased fourfold, the transmission risk is increased twofold. The compliance rate is limited by human nature, and therefore, if the prevalence of carriers becomes high, the chance of contact inevitably increases. The results of this formula suggest that, when many carriers exist in a ward, infection control practices may not be effective.

In terms of colonization, we need to consider the degree of environmental contamination surrounding carriers. As the degree of bacterial shedding from colonized patients increases, contamination of the environment increases; that is, the colonization pressure increases. Wang et $\mathrm{al}^{6}$ proposed that the colonization pressure is adjusted by the degree of environmental contamination. The modified colonization pressure, calculated by multiplying colonization pressure by the degree of environmental contamination, can be used for the formula. In addition, contamination is not limited to environmental surfaces. Infectious agents are transmitted via aerial droplet cloud, ${ }^{7,8}$ which could spatially or three-dimensionally enhance the colonization pressure and further spread infectious agents.

Colonization pressure could be defined as the total amount of infectious agents in an environment contaminated by carriers. The more rigorously that healthcare workers perform infection control measures, including hand hygiene and barrier precautions, the less easily infectious agents can be transmitted to patients. This phenomenon is similar to conditions in which resistance interrupts electric current. Infection control measures create resistance against infectious flow. If the total amount of infectious agents (ie, voltage) is high and infection control (ie, resistance) is low, the transmission (ie, current) becomes intense. Thus, the proposed formula could mimic Ohm's law regarding electric circuits, as follows: infectious current $=$ total amount of infectious agents in an environment/resistance to transmission to patients. In light of the formula, the measure of infection control can be divided into the 2 basic categories: colonization pressure (the numerator) and compliance rate (the denominator).

Controlling colonization pressure involves the following: early hospital discharge of colonized patients, isolation precautions, decolonization therapy, decreasing the quantity of 
contaminated surfaces (ie, cleaning and disinfecting of the environment), decreasing contact opportunities of contaminated surfaces using hands-free equipment (eg, nontouch thermometer, automatic faucet, and sensor-equipped room light), ventilation to dilute and remove contaminated air. However, increasing the compliance rate of hand hygiene and PPE measures by using various inventions, such as a bundle ${ }^{9}$ and checklist, improved access to PPE, ${ }^{10}$ and the development of a safety culture,$^{10}$ is also important. The proposed formula might describe the natural phenomenon of healthcare infection in a simple manner, but it could also be useful to effectively organize the historically accumulated knowledge of infection control and aid in the development of new strategies.

\section{ACKNOWLEDGMENTS}

Potential conflicts of interest. All authors report no conflicts of interest relevant to this article. All authors submitted the ICMJE Form for Disclosure of Potential Conflicts of Interest, and the conflicts that the editors consider relevant to this article are disclosed here.

\section{Kenji Kono, MD, FSHEA}

Affiliation: 1. Infection Control Department, Fukuoka Kinen Hospital, Fukuoka, Japan.

Address correspondence to Kenji Kono, MD, FSHEA, 1-1-35 Nishijin, Sawara-ku, Fukuoka, Japan 814-8525 (k-kono@hica.jp).

Infect Control Hosp Epidemiol 2014;35(9):1200-1201

(C) 2014 by The Society for Healthcare Epidemiology of America. All rights reserved. 0899-823X/2014/3509-0021\$15.00. DOI: 10.1086/677645

\section{REFERENCES}

1. Williams VR, Callery S, Vearncombe M, Simor AE. The role of colonization pressure in nosocomial transmission of methicillinresistant Staphylococcus aureus. Am J Infect Control 2009;37(2): 106-110.

2. Bonten MJ, Slaughter S, Ambergen AW, et al. The role of "colonization pressure" in the spread of vancomycin-resistant enterococci: an important infection control variable. Arch Intern Med 1998;158(10):1127-1132.

3. Merrer J, Santoli F, Appéré de Vecchi C, Tran B, De Jonghe B, Outin H. "Colonization pressure" and risk of acquisition of methicillin-resistant Staphylococcus aureus in a medical intensive care unit. Infect Control Hosp Epidemiol 2000;21(11):718-723.

4. Pavlov I. High colonization pressure might compromise the efficiency of routine methicillin-resistant Staphylococcus aureus screening. Clin Infect Dis 2007;44(5):766; author reply 766-767.

5. Pittet D, Hugonnet S, et al. Effectiveness of a hospital-wide programme to improve compliance with hand hygiene. Lancet. 2000;356(10):1307-1312.

6. Wang J, Wang M, Huang Y, et al. Colonization pressure adjusted by degree of environmental contamination: a better indicator for predicting methicillin-resistant Staphylococcus aureus acquisition. Am J Infect Control 201;39(9):763-769.

7. Eichenwald HF, Kotsevalov O, Fasso LA. The "cloud baby": an example of bacterial-viral interaction. Am J Dis Child 1960;100: 161-173.

8. Sherertz RJ, Bassetti S, Bassetti-Wyss B. "Cloud" health-care workers. Emerg Infect Dis 2001;7(2):241-244.

9. Muto CA, Blank MK, Marsh JW, et al. Control of an outbreak of infection with the hypervirulent Clostridium difficile BI strain in a university hospital using a comprehensive "bundle" approach. Clin Infect Dis 2007;45(10):1266-1273.

10. Yassi A, Lockhart K, Copes R, et al. Determinants of healthcare workers' compliance with infection control procedures. Healthc Q 2007;10(1):44-52.

\section{Epidemiology of Sharps Injury and Splash Exposure in an Oncology Care Center in Eastern India}

To the Editor-Sharps injury and splash exposure in the healthcare setting represent important occupational hazards and raise considerable concern about infection, psychological distress, and potential cost of management. Understanding the epidemiology of these incidents is essential for improved management strategies. In a 32-month period from May 2011 to January 2014, we report a total of 89 incidents of sharps injury and splash exposure from a 167-bed tertiary care oncology center in the eastern part of India. The male-to-female ratio was $1: 1.1$, and the age of the affected individuals ranged from 18 to 41.5 years for females (median age, 24.7 years) and from 19 to 47.2 years (median age, 25.9 years) for males. Staff distribution showed that 13 doctors, 38 nurses, 29 housekeeping staff, 6 laboratory workers, 2 operating room technologists, and 1 radiotherapy technologist were affected. The sharp injuries were associated with or caused by 54 hollow needles, 11 blades, 5 solid needles, 3 glass pieces, 2 diathermy devices, and 1 each of core biopsy needle, biopsy gun, microtome blade, and needle used for monitoring capillary blood glucose; 5 cases were associated with miscellaneous or unidentified sharps. Five $(5.6 \%)$ of 89 incidents involved splash exposures. An accelerated course of hepatitis B virus (HBV) vaccination (administered at day 0, 1 month, 2 months, and 12 months followed by postvaccination immunity testing at 6 weeks after the last dose of vaccine) was started for 43 (48\%) of 89 affected staff. However, vaccination uptake was not satisfactory in all cases; 11 (25\%) of 43 individuals received up to the fourth dose, 12 (38\%) of 43 received up to the third dose, and $5(12 \%)$ of 43 received 2 doses only. All staff (4 of 4 individuals) who were tested for hepatitis B surface antibody after the fourth dose had acquired satisfactory immunity $(\geq 10 \mathrm{mIU} / \mathrm{mL})$. Human immunodeficiency virus (HIV) postexposure prophylaxis or hepatitis $\mathrm{B}$ immunoglobulin were not required for any staff members $(0$ of 89). However, 7 staff members required tetanus prophylaxis because of soiling of wound and inadequate previous 